\title{
Research on Molecular Diffusion Coefficient of Gas-Oil System
}

\author{
Anping Ye1, Xiaoying Shen'2, Ping Guo', Shaoping Wang33, Zhongzhao Cheng4 \\ ${ }^{1}$ State Key Laboratory of Oil and Gas Reservoir Geology and Exploitation, Southwest Petroleum University, \\ Chengdu, China \\ ${ }^{2}$ State Intellectual Property Office, Beijing, China \\ ${ }^{3}$ No.2 Oil Production Plant, Petro China Changqing Oilfield Company, Qingyang, China \\ ${ }^{4} X i$ 'an Changqing Technology \& Engineering Company Ltd., Xi'an, China \\ Email: 156830216@qq.com
}

Published on Hans Journal of Chemical Engineering and Technology, Vol. 3, No. 2, 2013; available on http://www.hanspub.org/journal/PaperInformation.aspx?paperID=9601

\begin{abstract}
During the process of gas injection flooding, when the gas comes into contact with oil, the masstransfer phenomenon occurs. This changes the properties of the crude oil, improves the flow capacity, and thus enhances the oil recovery. Molecular diffusion coefficient is an important parameter to describe the mass-transfer rate of gas-oil system. At present, molecular diffusion coefficient is usually tested via indirect methods. There are 2 key points that should be noticed when testing this parameter. Firstly, select proper experimental methods. Secondly, use accurate model to describe the experimental data. In this research, the authors describe the researches on the experiment methods for determining diffusion coefficient for gas-oil system, the experiments and models on pressure decay, and the influencing factors that determine diffusion coefficient.
\end{abstract}

\section{Keywords}

Oil, Mass Transfer, Molecule Diffusion Coefficient 